01

\title{
Захват примеси в разбавленном растворе: фазово-полевое моделирование затвердевания
}

\author{
(C) А.А. Новокрещенова, ${ }^{1}$ В.Г. Лебедев, ${ }^{1,2}$ П.К. Галенко ${ }^{3,4}$ \\ ${ }^{1}$ Удмуртский государственный университет, \\ 426034 Ижевск, Россия \\ ${ }^{2}$ Научный центр МФМ УдмФИЦУрО РАН, \\ 426067 Ижевск, Россия \\ ${ }^{3}$ Йенский университет Фридриха Шиллера, физико-астрономический фракультет, \\ 07743 Йена, Германия \\ ${ }^{4}$ Лаборатория многомасштабного математического моделирования, Уральский фредеральный университет, \\ 620002 Екатеринбург, Россия \\ e-mail: Ivg@udsu.ru
}

Поступило в Редакцию 24 ноября 2020 г.

В окончательной редакции 4 декабря 2020 г.

Принято к публикации 4 декабря 2020 г.

На основе численного моделирования нестационарного процесса направленного затвердевания слабого раствора с плоским фронтом в рамках локально-неравновесной модели фазового поля выполнено исследование процесса перераспределения примеси в окрестности диффузной границы между жидкой и твердой фазами. В результате расчетов получены профили средней концентрации примеси вблизи межфазной границы. Из полученных профилей с помощью фазового поля выделены распределения концентраций в твердой и жидкой фазах при различных скоростях движения фронта. Построенные графики зависимости коэффициента распределения от скорости сравниваются с данными для гиперболической модели непрерывного роста и ранее выполненными расчетами в квазистационарном приближении. Проведено исследование влияния параметров модели на вид зависимости коэффициента распределения от скорости движения фронта и на профиль распределения концентрации внутри диффузной границы.

Ключевые слова: быстрые процессы затвердевания, локально-неравновесная ЕFКР-модель, коэффициент распределения.

DOI: 10.21883/JTF.2021.05.50685.324-20

\section{Введение}

Процессы литья до сих пор остаются одной из основных технологий обработки металлов. Независимо от вида литья, является ли оно классическим [1-3], типа литья в форму, или относится к продвинутым аддитивным технологиям [4], конечное качество металлического изделия во многом зависит от микроструктуры металла, формирующейся в процессе фазового перехода. Прогнозирование такой микроструктуры является актуальной задачей современного материаловедения. В силу сложности и нелинейности явления, в основе прогноза микроструктуры может лежать только компьютерное моделирование, аналогичное тому, как это делается в известных пакетах „Magma“, „ProCast“, „LVMFlow“ [5] для техпроцессов металлургии. К сожалению, внутренняя микроструктура металлов в этих пакетах совершенно не прогнозируется. Некоторый шаг в этом направлении был сделан разработчиками пакета „MICRESS“ [6]. Но дальнейшее развитие и разработка программных средств в данном направлении во многом лимитированы пониманием процесса распределения примеси на границах фаз. Поэтому в настоящее время продвижение в материаловедении связаны не столько с экспериментальными исследованиями, сколько с разработкой моделей затвердевания [7-9] и их верификации. Такое направление развития еще более актуально для аддитивных технологий, обеспечивающих высокие скорости охлаждения и большие температурные градиенты [10], что, в свою очередь, приводит к высоким скоростям движения границы раздела фаз в процессах плавления-кристаллизации. Именно при высоких скоростях движения межфазной границы становится актуальным понимание процесса захвата примеси, поэтому ее распределение вблизи границы активно исследуется в многочисленных работах $[11,12]$.

Сразу возникает вопрос об эталоне, на который стоит ориентироваться при такой верификации. Среди всех моделей затвердевания хорошо известна HCG-модель (Hyperbolic Continuous Growth model) роста [13], подтвержденная как экспериментом [14], так и моделированием методом молекулярной динамики [15]. Однако задача отслеживания резкой межфазной границы в трехмерном пространстве с течением времени математически весьма нетривиальна, из-за чего от такой постановки давно отказались на практике [5], предпочитая иметь дело с диффузной границей, т. е. границей, имеющей конечную протяженность в пространстве по направлению нормали. В зависимости от используемых масштабов 
описание диффузной границы носит название „теории двухфазной зоны“ [1-3] (применимой на макромасштабах $\sim 0.01-1 \mathrm{~m})$, либо „модели фазового поля“ $[16]$ (с характерной шириной границы, охватывающей мезомасштабы $\left.\sim 10^{-5}-10^{-8} \mathrm{~m}\right)$.

На сегодня наиболее перспективным направлением в компьютерном моделировании микроструктуры является идеология фазового поля. Среди различных моделей с идеологией фазового поля можно выделить модель Эчеваррии-Фолча-Карма-Плаппа (далее ЕFКР-модель) [17]. Модель была специально разработана с учетом возможных физических процессов на диффузных границах, правда только для разбавленных растворов. Поэтому естественно считать, что поведение именно этой модели должно воспроизводиться при дальнейшем развитии. Некоторые исследования модели EFKP были сделаны в работах $[18,19]$, но прямого численного моделирования ЕFКР до сих пор не было выполнено. Настоящая работа заполняет этот пробел, представляя результаты прямого численного моделирования процесса затвердевания и исследования влияния параметров модели EFКР на полученное решение.

\section{1. Коэффициент распределения примеси}

Процесс затвердевания бинарных растворов при слабом отклонении от равновесия сопровождается перераспределением примеси между твердой и жидкой фазами, что приводит к различным значениям концентраций в каждой из фаз, соответствующих равновесной диаграмме состояния $[1-3,20]$. Величина, равная отношению концентрации в твердой фазе к концентрации в жидкой фазе на границе раздела жидкость-твердая фаза, носит название коэффициента распределения $k$ :

$$
k=\frac{C_{S}}{C_{L}},
$$

значения которого экспериментально определены для различных веществ [21] в широком интервале переохлаждений и скоростей движения фронта затвердевания. $C_{S}, C_{L}$ - соответствующие значения концентраций примеси на границе фаз. Особенно подробно коэффициент распределения исследовался для системы $\mathrm{Si}-\mathrm{As}$ [22-24]. Поэтому зависимость коэффициента распределения $k(V)$ от скорости движения фронта для системы $\mathrm{Si}-\mathrm{As}$ служит тестовой задачей, позволяющей сравнивать между собой различные модели затвердевания $[15,18,19,25-31]$.

Проведенные экспериментальные исследования и численные расчеты показывают [21], что увеличение степени неравновесности в системе (увеличение переохлаждения системы по отношению к ликвидусу и соответственно скорости движения фронта затвердевания) приводит к обращению $k(V)$ в единицу, что интерпретируется как полный „захват примеси“ твердой фазой („solute trapping“). Из расчетов следует, что в рамках локальноравновесных подходов $[27,32]$ соответствующее поведение $k(V)$ является асимптотическим, в то время как для локально-неравновесных подходов существует критическая скорость движения фронта $V=V_{D}$ превышение которой приводит к бездиффузионному затвердеванию, для которого коэффициент распределения обращается в единицу $\left(k(V)=1\right.$ при $\left.V \geq V_{D}\right)$ [33]. Подобная зависимость впоследствие нашла подтверждение в компьютерных экспериментах на основе молекулярной динамики [15]. Процессы, в которых переохлаждение системы намного ниже температуры ликвидуса [21], называют процессами быстрого затвердевания, поскольку для них движение границы раздела твердое тело-жидкость происходит со скоростью, сравнимой с характерной скоростью $V \approx V_{D}$.

\section{2. Фазовое поле}

Большинство упомянутых моделей связано с фазово-полевым подходом [16], в котором для описания среды вводится нужное число параметров порядка, каждое из которых принимает значение единица в одной из фаз и нуль в остальных. Такие параметры порядка называются фазовыми полями, которые, по сути, обобщают теорию двухфазной зоны, позволяя более существенно использовать неравновесную термодинамику и применять модели на мезоскопических масштабах $\left(10^{-7}-10^{-8} \mathrm{~m}\right)$. Исследование процесса перераспределения примеси остается актуальным вопросом для метода фазового поля, позволяющего не только описывать сложную пространственную геометрию микроструктурных областей в процессе затвердевания, но и учесть сильнонеравновесные условия протекания процесса [34].

Численное моделирование процесса быстрой кристаллизации в квазистационарном подходе $[18,19]$ демонстрирует наличие характерного выступа („spike“) по концентрации внутри диффузной границы. Экспериментально до сих пор нет однозначного ответа на вопрос о существовании спайка. Действительно, с точки зрения эксперимента, задача определения концентраций на фронте фаз является вызовом к искусству экспериментатора и методике эксперимента. Но можно предположить, что появление спайка есть просто теоретический артефакт, поскольку причины появления выступа могут состоять как в выборе метода решения (квазистационарное приближение), так и в свойствах модели (выборе единой для обеих фаз функции концентрации, по которой с помощью фазового поля выделяются концентрации в отдельных фазах). Непосредственное интегрирование динамических уравнений модели $[18,19]$ ранее не использовалось из-за отсутствия численных алгоритмов для сингулярно-возмущенных уравнений, описывающих процессы затвердевания в локально-неравновесной модели. Градиентно-устойчивые алгоритмы интегрирования соответствующих уравнений предложены в работах $[35,36]$. 
Более конкретно цели работы состоят в приложении алгоритмов $[35,36]$ к задачам моделирования процессов быстрого затвердевания, в исследовании причин появления выступа по концентрации на диффузной границе, а также в исследовании влияния параметров модели на выступ профиля концентрации внутри диффузной границы и на коэффициент распределения примеси.

В качестве исследуемой модели выбрано локальнонеравновесное обобщение ЕFКР-модели [19], позволяющее учитывать произвольные переохлаждения разбавленного бинарного расплава. Локально-неравновесная модель ЕFКР является частным случаем фазово-полевого подхода к описанию фазовых переходов и представлена системой гиперболических нелинейных уравнений в частных производных. Исходная локально-равновесная версия ЕFКР-модели [17] была разработана с целью моделирования процесса формирования микроструктуры при направленном затвердевании разбавленного бинарного расплава с малым переохлаждением. Преимущество ЕFКР-модели [17] по сравнению с предыдущими моделями фазового поля [25-28,32] состоит в учете „компенсирующего“ потока примеси (,antitrapping“) в законе сохранения вещества [37]. Этот поток корректирует увеличение эффекта захвата примеси в случае, когда характерная толщина диффузной границы между фазами искусственно завышается при компьютерном моделировании процесса затвердевания с целью повышения производительности расчетов. Кроме того, введение „компенсирующего“ потока обеспечивает дополнительную свободу в модели ЕFКР. Эта свобода позволяет подавить ложные эффекты на масштабах диффузной границы, возникающие из-за неравенства коэффициентов диффузии в твердой и жидкой фазах [38]. Сравнительный анализ ЕFКР-модели по сравнению с ранее используемыми фазово-полевыми моделями затвердевания [25-28,32] можно найти в [19].

Для анализа перераспределения примеси в рамках EFКР-модели выполнено компьютерное моделирование нестационарной задачи затвердевания для системы $\mathrm{Si}-\mathrm{As}$ с плоским фронтом. Поскольку в рамках EFКР-модели предполагается, что функция концентрации примеси является единой для всех фаз, для определения зависимости коэффициента распределения от скорости движения границы использовано два разных определения: по максимумам в жидкой и твердой фазах [19] и по границам диффузной зоны [18].

\section{3. Численная постановка краевой задачи}

Моделирование задачи направленного затвердевания с плоским фронтом предполагает решение одномерных уравнений в частных производных, описывающих процесс затвердевания, на бесконечном интервале при заданном переохлаждении ( $T=\mathrm{const}$, причем температура меньше температуры ликвидуса при заданной кон- центрации $T<T_{m}-m_{e} C, m_{e}-$ наклон линии ликвидуса на равновесной фазовой диаграмме) и выход решения на установившийся режим, при котором происходит сдвиг фазовой границы с постоянной скоростью. Будем полагать, что направление движения фронта совпадает с положительным направлением оси $x$. Для определенности примем, что жидкой фазе соответствует $\varphi=1$, а твердой $-\varphi=0$. Тогда безразмерные уравнения модели (см. Приложение, П8, П10) имеют вид

$$
\begin{aligned}
& \widetilde{\tau}_{\varphi} \frac{\partial^{2} \varphi}{\partial t^{2}}+\frac{\partial \varphi}{\partial t}= \frac{\partial^{2} \varphi}{\partial x^{2}}-\frac{9}{2} \frac{\partial g(\varphi)}{\partial \varphi} \\
&+\frac{1}{2} \frac{\delta}{d_{0}} \frac{T}{T_{A}} \lambda(T, C, \varphi) \frac{\partial p(\varphi)}{\partial \varphi}, \\
& \widetilde{\tau}_{D} \frac{\partial^{2} C}{\partial t^{2}}+\frac{\partial C}{\partial t}=\frac{\partial}{\partial x}\left(\widetilde{D}(\varphi) \frac{\partial C}{\partial x}\right) \\
&+\frac{\partial}{\partial x}\left(\Theta(T) \widetilde{D}(\varphi) C \frac{\partial p(\varphi)}{\partial \varphi} \frac{\partial \varphi}{\partial x}\right),
\end{aligned}
$$

где

$$
\lambda(T, C, \varphi)=\frac{\left(1-k_{e}\right) C}{k_{e}+\left(1-k_{e}\right) p(\varphi)}-\frac{1-k_{e}}{m_{e}}\left(T-T_{A}\right),
$$

$\tilde{\tau}_{\varphi}$ - безразмерное время релаксации скорости изменения фазового поля, $d_{0}-$ капиллярная длина, $k_{e}-$ коэффициент перераспределения, $\delta$ - толщина границы раздела, $T_{A}$ - температура плавления основного компонента функции $p(\varphi), g(\varphi), \Theta(T)$ определены как

$$
\begin{gathered}
p(\varphi)=\varphi^{2}(3-2 \varphi), \\
g(\varphi)=\varphi^{2}(1-\varphi)^{2}, \\
\Theta(T)=-\frac{1-k_{e}}{k_{e}+\left(1-k_{e}\right) p(\varphi)},
\end{gathered}
$$

$\widetilde{\tau}_{D}$ - безразмерное время релаксации потока примеси, $\widetilde{D}_{\varphi}$ - безразмерный коэффициент диффузии. В качестве начального условия для фазового поля используем стационарное решение:

$$
\varphi\left(x, t_{0}\right)=\frac{1}{2}\left(1+\tanh \left(\frac{3(x-2 \delta)}{2 \delta}\right)\right) .
$$

В предположении, что начальное распределение концентрации переохлажденного раствора является однородным, начальное условие для концентрационного поля (в мольных долях) задано постоянным $C\left(t_{0}\right)=C_{0}=0.001$ (для сравнения с результатами работы [19]). Материальные параметры модели также взяты из работы [19] и приведены в (см. Приложение).

Для перехода к расчетам на конечном интервале, чтобы избежать искажений решения вблизи границы, правая граница области, к которой со временем приближается фронт кристаллизации, должна смещаться с течением времени. В силу однородности системы, 
при численном решении задачи удобнее сделать обратную операцию: решение транслируется в направлении, противоположном направлению движения фронта, когда фронт приближается к правой границе на определенное расстояние. Используемые граничные условия для задачи на конечном интервале имеют вид

$$
\begin{gathered}
\left.\varphi\right|_{x=0}=1,\left.\quad \frac{\partial C}{\partial x}\right|_{x=0}=0, \\
\left.\frac{\partial \varphi}{\partial x}\right|_{x=L}=0,\left.\quad \frac{\partial C}{\partial x}\right|_{x=L}=0 .
\end{gathered}
$$

Для численного решения гиперболических уравнений (2), (3), с учетом того что фазовое поле $\varphi$ является несохраняющейся, а концентрация $C-$ сохраняющейся величиной, получены полунеявные градиентноустойчивые разностные схемы [35,36], представленные в приложении (П14-П17). Там же обсуждаются устойчивость и точность используемого алгоритма.

\section{4. Определение скорости фронта и коэффициента распределения $k(V)$}

В силу того что профиль фазового поля в любой момент времени слабо отличается от исходного кинка (8), положение фронта, т.е. точка такая, что может быть приближенно найдено, как

$$
x_{0}=\int_{0}^{L_{0}}(1-\varphi(x)) d x,
$$

где $L_{0}-$ пространственный размер области, в которой ищется решение. Скорость движения фронта определялась как средняя скорость движения по $N$ временным шагам

$$
V=\frac{x\left(t_{0}+N \Delta t\right)-x\left(t_{0}\right)}{N \Delta t} .
$$

Поскольку коэффициент распределения $k(V)$ в случае диффузной границы и единой функцией концентрации для обеих фаз не является однозначно определенной величиной, использовалось два подхода:

1. В соответствии с [19] для определения коэффициента перераспределения по максимумам в жидкой и твердой фазах концентрационное поле $C(x)$ представляется через концентрацию раствора в жидкой $C_{L}$ и твердой $C_{S}$ фазах как

$$
C(x)=C_{L}(x)+C_{S}(x)
$$

где

$$
\begin{gathered}
C_{S}(x)=[1-h(p(\varphi))] C(x), \\
C_{L}(x)=h(p(\varphi)) C(x) .
\end{gathered}
$$

Функция $h(p(\varphi))$ обеспечивает плавное поведение функций $C_{L}$ и $C_{S}$ и имеет вид

$$
h(p)=\frac{p(\varphi)}{k(V)+(1-k(V)) p(\varphi)}
$$

где коэффициент перераспределения выбирается следующим соотношением:

$$
k(V)=\frac{\max \left(C_{S}(x)\right)}{\max \left(C_{L}(x)\right)} .
$$

2. Определение коэффициента перераспределения по границам диффузной зоны в соот- ветствии с [18] задается отношением

$$
k(V)=\frac{C_{S}}{C_{L}}=\frac{\left.C\right|_{\varphi=0.001}}{\left.C\right|_{\varphi=0.999}} .
$$

\section{5. Численное моделирование и концентрационные профили}

Характерные профили распределения примеси приведены на рис. 1. На рис. 1, $a$ представлены профили

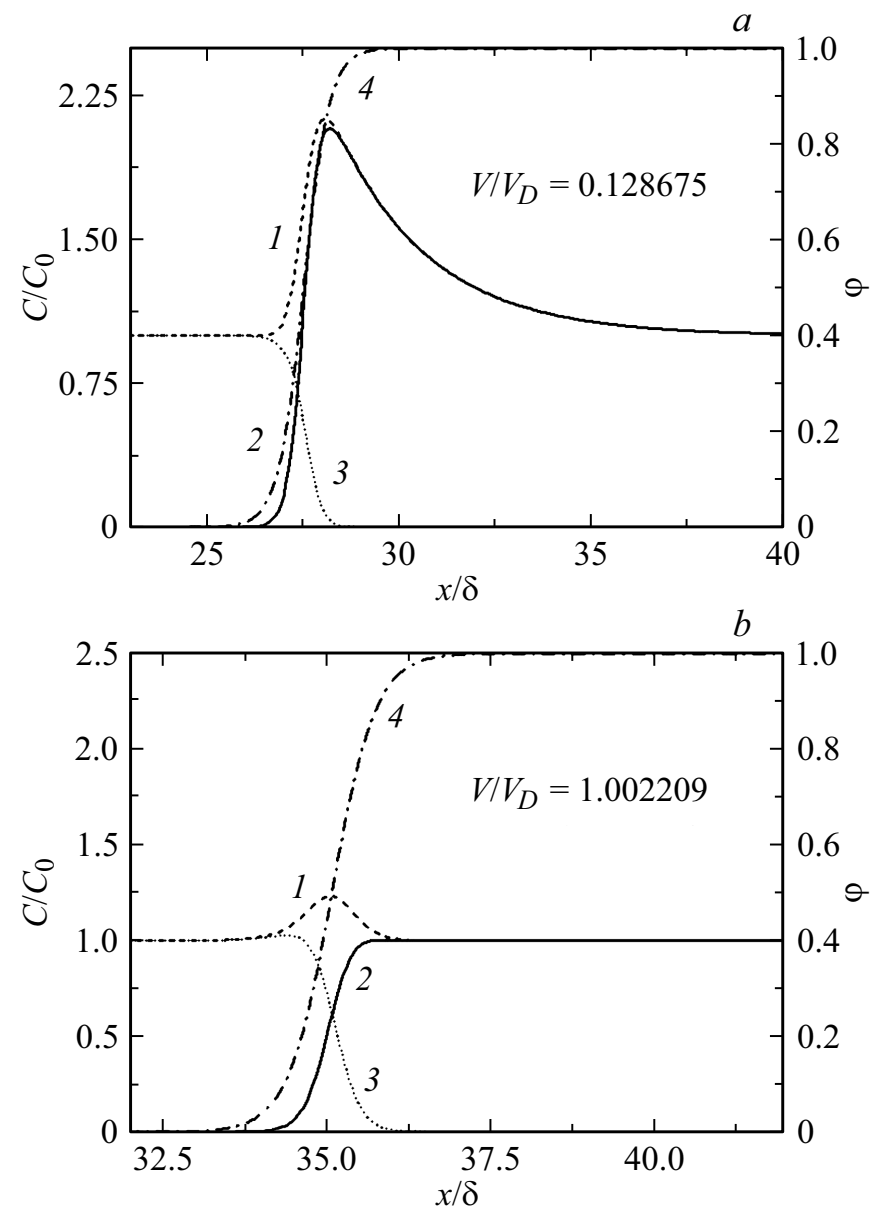

Рис. 1. Характерный вид профилей концентрации примеси $C / C_{0}: a-$ при малом переохлаждении; $b-$ при большом переохлаждении. Концентрации показаны: сплошной линией в жидкой фазе, крупным пунктиром - средняя по фазам концентрация, мелким пунктиром - концентрация в твердой фазе, штрихпунктирная линия соответствует фазовому полю. Выступ („spike“) для средней концентрации на диффузной границе сохраняется при $V / V_{D}>1$. 


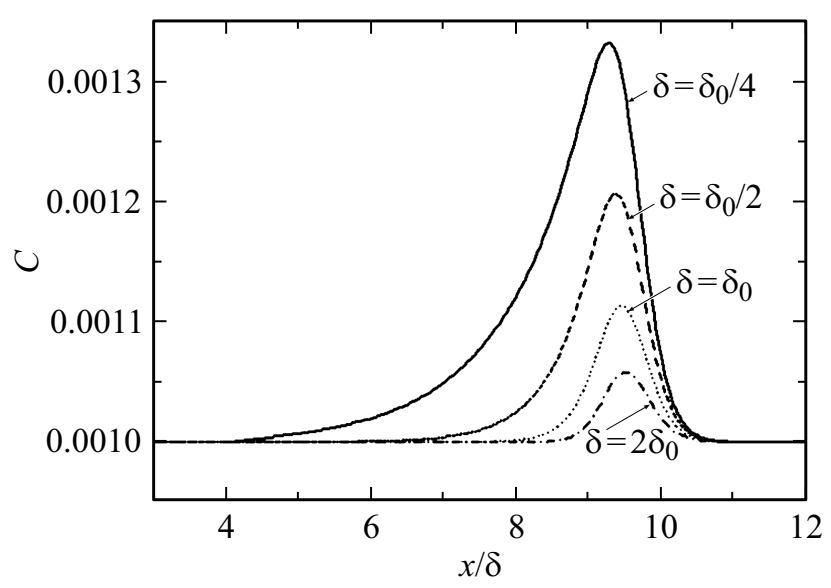

Рис. 2. Величина концентрационного выступа (концентрация $C$, атомные доли) в зависимости от безразмерной координаты $x / \delta$ при разной ширине границы $\delta$.

средней по фазам концентрации примеси, и ее проекции на твердую и жидкую фазы $C_{L}$ и $C_{S}$ при достаточно малой $\left(V / V_{D}=0.128675\right)$ скорости движения фронта. Движение фронта приводит к вытеснению избытка примеси в жидкую фазу, что проявляется на графике $C(x) / C_{0}$ в виде выступа, размытого по жидкой фазе за счет диффузии.

На рис. 1,b показаны профили средней по фазам концентрации примеси и ее проекции на твердую и жидкую фазы $C_{L}$ и $C_{S}$ при большой скорости движения фронта $V / V_{D}=1.002209$. Концентрационный спайк на графике стал более симметричным, чем в случае малых скоростей, и помещается внутри диффузной границы. Но полностью он не исчезает ни при каких переохлаждениях.

Более того, моделирование с различными значениями ширины диффузной границы показывают зависимость концентрационного выступа от ширины диффузной границы $\delta$. Эта зависимость показана на рис. 2, откуда видно, что величина выступа стремится к нулю лишь асимптотически в пределе $\delta \gg \delta_{0}$. На рис. 2 также видно, что при значениях $\delta \geq \delta_{0}$ начинает проявляться ассимметрия концентрационного выступа.

\section{6. Численные результаты для $k(V)$}

Для нахождения коэффициента распределения по максимумам в различных фазах были использованы итерации по соотношениям (15), (16). На первом шаге предполагалось равенство $k(V)=k_{e}$ с последующим уточнением результата до $\Delta k<10^{-15}$. Для вычисления коэффициента распределения по границам диффузной зоны применялся метод установления, при котором флуктуации средней скорости движения фронта не превышали величину $\Delta V \sim 10^{-9}$. Полученные зависимости коэффициента распределения от скорости движения границы для двух определений при ширине диффузной зоны $\delta_{0}$ и времени релаксации $\tau_{D}^{0}$ приведены на рис. 3 . Поскольку экспериментальные данные по коэффициенту распределения для используемого значения концентрации $C_{0}$ отсутствуют, результаты по ЕFКР-модели сравниваются с расчетами по гиперболической модели непрерывного роста HCG.

Из графиков рис. 3 видно, что два способа определения $k(V)$ в модели ЕFКР дают несколько отличающийся от HCG-модели результат, хотя очень близки друг к другу при больших и малых скоростях движения фронта. Хотя в целом графики для EFKР проходят близко к графику HCG-модели, отметим, что графики EFKP

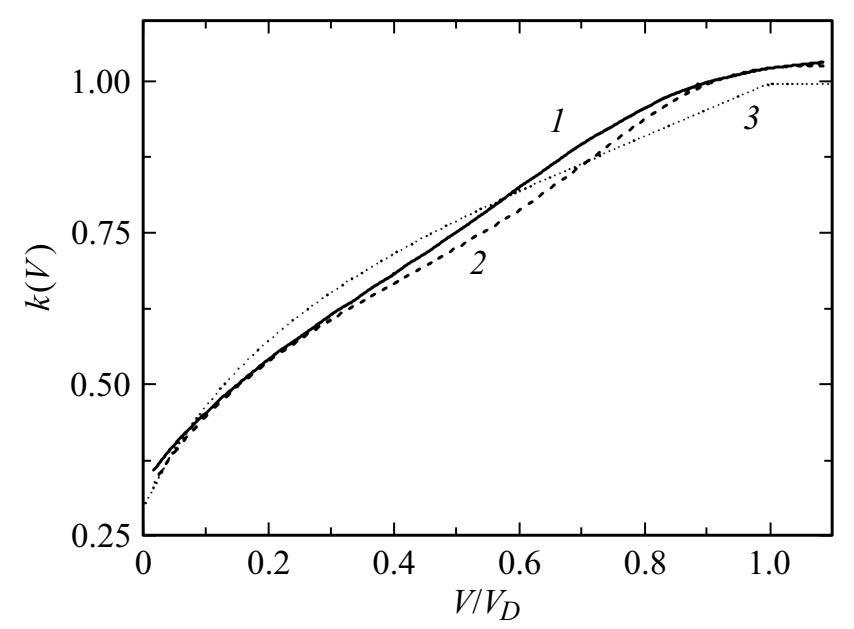

Рис. 3. Зависимость коэффициента распределения $k(V)$ от скорости движения фронта $V / V_{D}$ при определении по максимумам и по значениям на границе, а также сравнение с HCG-моделью. 1 - гиперболическая EFКР с определением $k(V)$ по максимумам, 2 - гиперболическая ЕFКР с определением $k(V)$ по диффузной границе, 3 - HCG-модель [13].

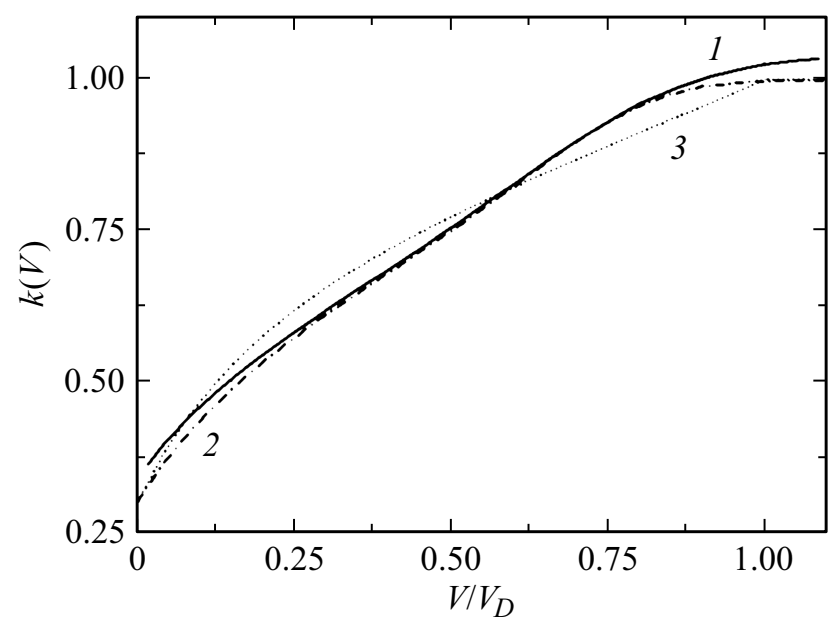

Рис. 4. Коэффициент распределения $k(V)$, вычисленный по максимумам концентраций в нестационарном подходе (настоящая работа), по сравнению с квазистационарным подходом [19]. 1 - гиперболическая ЕFКР с определением $k(V)$ по максимумам, 2 - гиперболическая EFKP [19], 3 - HCG-модель [13]. 


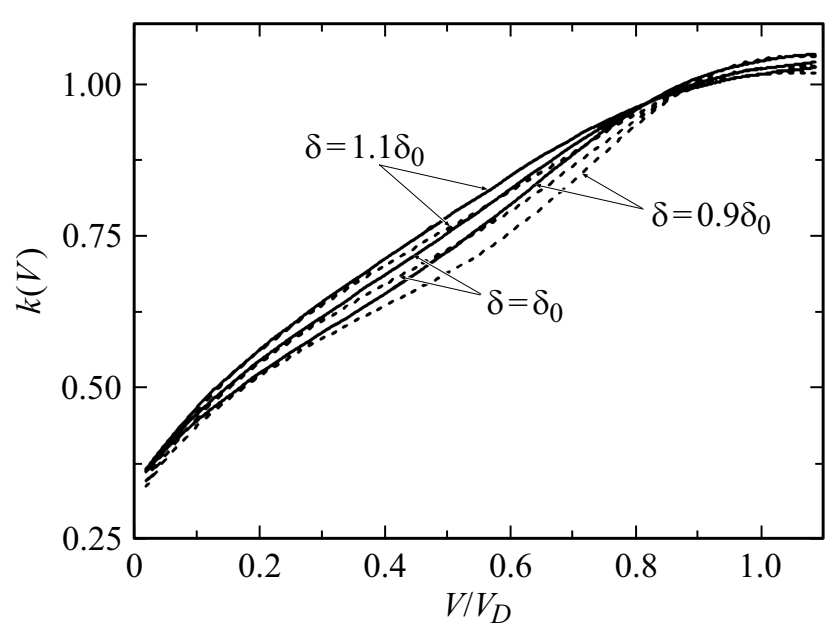

Рис. 5. Влияние ширины диффузной границы $\delta$ на вид коэффициента распределения $k(V)$. Сплошным линиям соответствует определение $k(V)$ по максимумам, пунктир - определение $k(V)$ по диффузной границе.

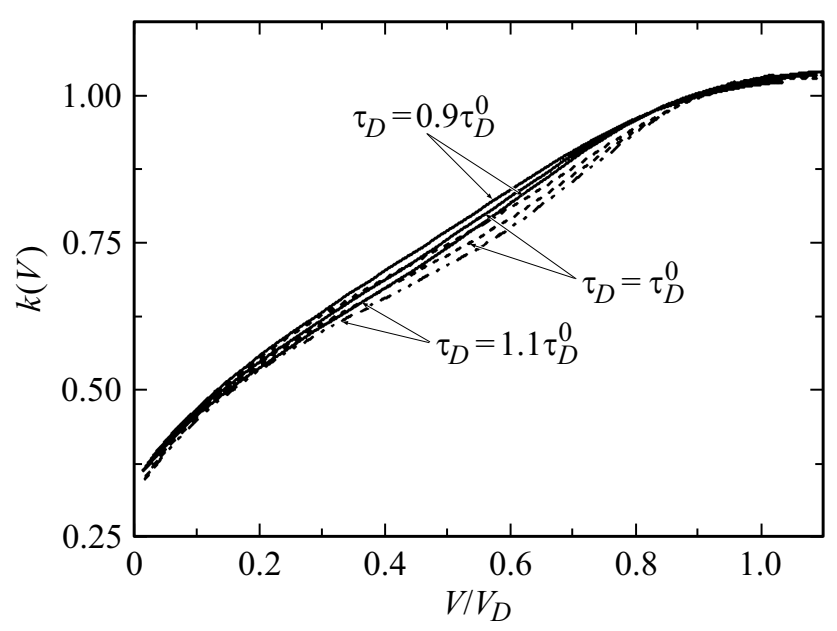

Рис. 6. Влияние времени релаксации $\tau_{D}$ на вид коэффициента распределения $k(V)$. Сплошным линиям соответствует определение $k(V)$ по максимумам, пунктир - определение $k(V)$ по диффузной границе.

пересекают $k(V)=1$ при меньших значениях скорости фронта, чем график HCG-модели.

Также отметим, что коэффициент распределения $k(V)$ вычисленный по максимумам концентраций при моделировании нестационарного процесса в настоящей работе, близок к графику, полученному в квазистационарном подходе [19] (рис. 4). Существенная разница проявляется при где видно, что график из работы [19] плавно выходит на горизонтальную асимптотику, а график, полученный в настоящей работе пересекает $k=1$. На графике рис. 4 также отчетливо видна разница между $k(V)$ для квазистационарного и нестационарного подходов при малых скоростях фронта. Эта разница свидетельствует либо о недостаточном времени релаксации нестационарного решения, либо о влиянии границы, поскольку „хвост“ распределения примеси, вытесненной в жидкую фазу, является протяженным и релаксирует медленно.

Поскольку процесс перераспределения примеси при затвердевании контролируется диффузией, в настоящей работе варьировались параметры, изменение которых могло повлечь за собой отличие процесса переноса примеси в рамках фазово-полевой модели (ЕFКР) от задачи с резкой границей (HCG-модель). В качестве таковых параметров рассматривались время релаксации диффузионного потока $\tau_{D}$ и ширина диффузной границы $\delta$. Их влияние на коэффициент распределения $k(V)$ представлено на рис. 5, 6. На обоих графиках видно, что изменение параметров влияет на наклон графиков внутри интервала $0<k(V)<1$, но значение скорости, при которой $k(V)=1$, практически не меняется.

\section{7. Обсуждение результатов}

Из результатов численного моделирования ЕFКР-модели можно сделать вывод о хорошем совпадении (с учетом необходимости более точных расчетов на малых скоростях движения фронта) результатов моделирования нестационарной задачи затвердевания и квазистационарного подхода, использованного в работе [19].

Основная проблема с сопоставлением результатов работы [19] и настоящих расчетов состоит в понимании двух фактов:

- нестационарное решение выходит на значение $k=1$ при скоростях движения фронта меньших, чем $V / V_{D}=1$;

- график $k(V)$ для нестационарного решения пересекает $k=1$ трансверсально, в то время как стационарное решение выходит на $k=1$ асимптотически, а HCG-модель предсказывает в точке $V / V_{D}$ резкое изменение поведения $k(V)$ характеризуемое скачком производных.

Относительно первого факта можно сказать следующее: такой результат обусловлен разницей в кинетике моделей, а именно меньшим значением скорости диффузии $V_{D}^{\varphi}$ в объеме для фазово-полевой модели: $V_{D}^{(\varphi)}=(1-p(\varphi)) V_{D}^{(L)}+p(\varphi) V_{D}^{(S)}$, где $V_{D}^{(L)}=$ $=V_{D}=\sqrt{D_{L} / \tau_{D}^{0}}-$ скорость диффузии в объеме жидкости и $V_{D}^{(S)}=\sqrt{D_{S} / \tau_{D}^{0}}<V_{D}-$ скорость диффузии в объеме твердой фазы (см. таблицу в Приложении). Действительно, с увеличением $V$ имеем $p(\varphi) \rightarrow 1$ и $V_{D}^{\varphi} \rightarrow V_{D}^{S}<V_{D}$. Поэтому переход к бездиффузионному затвердеванию в фазово-полевой модели происходит при меньшей скорости, чем в HCG-модели. Возможность скомпенсировать различие между HCG-моделью и неравновесной ЕFКР-моделью за счет изменения внутренних параметров оказывается ограниченной другим явлением: сильные изменения профиля концентрации при изменении ширины диффузной границы на рис. 2 говорят о том, что свойства поверхности раздела и объемные свойства в неравновесной ЕFКР-модели влияют 


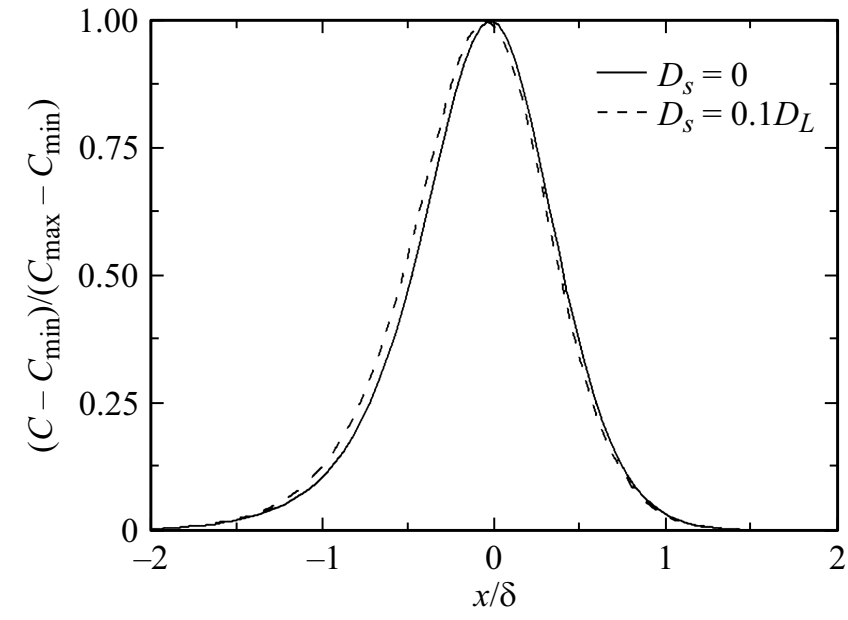

Рис. 7. Влияние соотношения между коэффициентами диффузии на симметрию концентрационного выступа („spike“) внутри и вблизи диффузной границы. Показано нормированное распределение концентраций вдоль безразмерной координаты $x / \delta$ при двух соотношениях коэффициентов диффузии.

друг на друга настолько, что это влияние не позволяет менять, например, ширину диффузной границы произвольным образом.

Второй факт - переход $k(V)$ через единицу трансверсальным образом, что приводит к формированию твердой фазы с концентрацией, превышающей концентрацию в жидкости. Такое поведение указывает на формирование неустойчивой твердой фазы. Для гиперболической ЕFКР-модели такой факт сигнализирует о недостаточной точности ее формулировки. В частности, это означает, что в ЕFКР-модели и более ранних моделях затвердевания [25-28] недостаточно точно описан механизм перераспределения примеси для высоких скоростей кристаллизации, что связано с дополнительным потоком в уравнении (П.4) (второе слагаемое с правой стороны). Из численных расчетов можно заключить, что ассимметрия графиков выступов на концентрационных профилях на рис. 3 определяется ассимметрией зависимости $D(\varphi)$, что в конечном итоге приводит к значениям коэффициента распределения $k>1$. На рис. 7 представлен вид нормированных профилей концентрации при коэффициентах диффузии $D_{S}=0$ и $D_{S}=0.1 D_{L}$ относительно положения диффузной границы между фазами $(x=0$ соответствует $\varphi=1 / 2)$. Из рисунка видно, что даже при $D_{S}=0$ профиль концентрации не симметричен относительно $x=0$. Дальнейшее увеличение диффузии в твердой фазе только увеличивает ассимметрию выступа („spike“), а последующее вычисление концентраций в фазах по правилам (15), (16) приводит к неправильным значениям $k(V)$. Таким образом, в рамках моделей, использующих единое описание концентраций во всех фазах, наличие „spike“, по-видимому, является наследственным артефактом, присущим всему семейству моделей. Так что дело остается за экспериментом по измерению концентраций на масштабах диффузной границы.

\section{Выводы}

Основной вывод, который следует из прямого численного моделирования ЕFКР- модели, состоит в том, что модель имеет ряд преимуществ как по сравнению с предшествующими фазово-полевыми моделями затвердевания [25-28], которые до сих пор используются для изучения микроструктуры материалов $[39,40]$, так и по сравнению с новыми моделями [7-9]. Во-первых, по сравнению с известной моделью Уилера-Беттингера-Макфаддена [25,26] наблюдается более правильное поведение коэффициента распределения, хотя ЕFКР-модель тоже нуждается в доопределении, позволяющем решить проблему с возникновением $k(V)>1$. Но до этого предельного значения ЕFКР-модель вполне адекватно описывает перераспределение примеси на границе. Несомненно, модель ЕFКР имеет важное преимущество перед уже упомянутыми моделями [7-9] в силу своей простоты. Последнее позволяет произвести достаточно полный анализ различных решений в модели подобно работам $[41,42]$, подсказав возможную модификацию ЕFКР при больших скоростях не только за счет локальной неравновесности.

\section{Приложение}

\section{1. Уравнения EFKР-модели затвердевания разбавленного бинарного раствора}

Для анализа захвата примеси при быстрой кристаллизации рассмотрим бинарную систему, состоящую из атомов сорта $A$ (растворитель), а также небольшого количества атомов сорта $B$ (примесь) при постоянной температуре $T$ и постоянном давлении, принятом за нуль. Динамика переменных определяется функционалом свободной энергии локально-равновесной системы вида:

$$
F=\int_{\Omega}\left(f_{e q}(C, \varphi)+\frac{1}{2} \varepsilon_{\varphi}^{2}(\nabla \varphi)^{2}\right) d \Omega,
$$

где $\Omega$ - пространственная область, занятая системой, а плотность равновесной свободной энергии в случае слабого раствора для модели ЕFКР выбрана в виде

$$
\begin{aligned}
f_{e q}(C, \varphi)= & f^{A}\left(T_{A}\right)-\left(T-T_{A}\right) s(\varphi)+\epsilon(\varphi) C \\
& +\frac{R T}{v_{m}}(C \ln C-C)+W_{g}(\varphi) .
\end{aligned}
$$

В выражениях (П1), (П2) использованы обозначения $\varepsilon_{\varphi}^{2}=2 \sigma \delta-$ параметр для градиентного слагаемого энергии, где $\sigma$ - энергия границы раздела, $\delta$ - толщина 
Параметры фазового поля для расплава $\mathrm{Si}-0.1$ at.\% As [19]

\begin{tabular}{|c|c|c|}
\hline Параметр & Обозначение & Численное значение \\
\hline $\begin{array}{l}\text { Молярный объем } \\
\text { Коэффициент диффузии в жидкости } \\
\text { Коэффициент диффузии в твердой фазе } \\
\text { Коэффициент поверхностного натяжения } \\
\text { Мобильность фазового поля } \\
\text { Кинетический коэффициент фазового поля } \\
\text { Ширина диффузной границы } \\
\text { Газовая постоянная } \\
\text { Время релаксации фазового поля } \\
\text { Время релаксации потока примеси } \\
\text { Температура плавления чистого компонента } \\
\text { Наклон линии ликвидуса } \\
\text { Равновесный коэффициент распределения }\end{array}$ & $\begin{array}{c}v_{m} \\
D_{L} \\
D_{S} \\
\sigma \\
M_{\varphi} \\
\nu \\
\delta_{0} \\
R \\
\tau_{\varphi} \\
\tau_{D}^{0} \\
T_{A} \\
m_{e} \\
k_{e}\end{array}$ & $\begin{array}{c}1.2 \cdot 10^{-5} \mathrm{~m}^{3} / \mathrm{mol} \\
1.5 \cdot 10^{-9} \mathrm{~m}^{2} / \mathrm{s} \\
3 \cdot 10^{-13} \mathrm{~m}^{2} / \mathrm{s} \\
0.477 \mathrm{~J} / \mathrm{m}^{2} \\
8.777 \mathrm{~m}^{3} /(\mathrm{J} \cdot \mathrm{s}) \\
1.22 \cdot 10^{-8} \mathrm{~m}^{2} / \mathrm{s} \\
1.875 \cdot 10^{-9} \mathrm{~m} \\
8.314 \mathrm{~J} /(\mathrm{mol} \cdot \mathrm{K}) \\
10^{-11} \mathrm{~s} \\
2.4 \cdot 10^{-10} \mathrm{~s} \\
1685 \mathrm{~K} \\
-400 \mathrm{~K} \\
0.3\end{array}$ \\
\hline
\end{tabular}

границы раздела, $f^{A}\left(T_{A}\right)$ соответствует свободной энергии чистой системы растворителя (компонент $A$ ), $T_{A}-$ температура плавления чистого компонента $A, R-$ универсальная газовая постоянная, $v_{m}$ - мольный объем, $W=9 \sigma / \delta$ - высота энергетического барьера, который моделируется функцией вида $g(\varphi)=\varphi^{2}(1-\varphi)^{2}$.

Плотность энтропии $s(\varphi)$ и внутренняя плотности энергии $\epsilon(\varphi)$ получены с использованием приближения разбавленного раствора

$$
\begin{gathered}
s(\varphi)=\frac{s_{s}+s_{i}}{2}-p_{s}(\varphi) \frac{L}{2 T_{A}}, \quad p_{s}(\varphi)=1-2 p(\varphi), \\
\epsilon(\varphi)=\frac{\epsilon_{s}+\epsilon_{i}}{2}-p_{\epsilon}(\varphi) \frac{R T}{2 v_{m}} \ln k_{e} \\
p_{\epsilon}(\varphi)=\frac{2}{\ln k_{e}} \ln \left[k_{e}+p(\varphi)\left(1-k_{e}\right)\right]-1,
\end{gathered}
$$

где $L=-R T\left(1-k_{e}\right) T_{A} /\left(v_{m} m_{e}\right)$ - скрытая теплота затвердевания, $k_{e}-$ коэффициент перераспределения, $m_{e}$ - наклон линии ликвидуса на равновесной фазовой диаграмме, индексы $s$ и $i$ связаны с твердой и жидкой фазами соответственно, а функция $p(\varphi)$ определена как $p(\varphi)=\varphi^{2}(3-2 \varphi)$. Отметим, что функция $p(\varphi)$ является монотонной в интервале $[0,1]$ и удовлетворяет уравнениям $p_{\varphi}^{\prime}(0)$ и $p_{\varphi}^{\prime}(1)=0$ в соответствующих фазах, что является необходимым условием устойчивости термодинамических фаз [43].

Требование монотонного убывания функционала свободной энергии, учитывающего локально-неравновесные вклады [34] от изменения потоков, по мере релаксации системы к равновесию приводит к уравнениям динамики для концентрации и фазового поля [19]:

$$
\begin{gathered}
\tau_{D} \ddot{C}+\dot{C}=\nabla\left[M_{G}\left(\frac{\partial^{2} f_{e q}}{\partial C^{2}}+\frac{\partial^{2} f_{e q}}{\partial C \partial \varphi}\right)\right], \\
\tau_{\varphi} \ddot{\varphi}+\dot{\varphi}=M_{\varphi}\left(\varepsilon_{\varphi}^{2} \Delta \varphi-\frac{\partial f_{e q}}{\partial \varphi}\right)
\end{gathered}
$$

где $\tau_{D}$ - время релаксации потока примеси, $M_{G}-$ коэффициент мобильности для $B$-атомов, $\tau_{\varphi}-$ время релаксации скорости изменения фазового поля и $M_{\varphi}-$ коэффициент мобильности фазового поля. Тогда при условии положительности коэффициентов мобильности уравнения (П4), (П5) гарантируют невозрастание свободной энергии и выполнение закона сохранения вещества для примеси.

\section{2. Безразмерные уравнения модели}

Для получения уравнений модели в явном виде воспользуемся формулами (П4), (П5), подставив в них производные плотности свободной энергии и выразив коэффициент концентрационной мобильности через коэффициенты диффузии в фазах

$$
M_{G}(T, C, \varphi)=\left(\frac{\partial^{2} f}{\partial C^{2}}\right)^{-1} D(\varphi)=\frac{v_{m} C}{R T} D(\varphi),
$$

где $D(\varphi)=D_{S}+p(\varphi)\left(D_{L}-D_{S}\right)$ - интерполяционная зависимость коэффициента диффузии, $D_{L}, D_{S}-$ коэффициенты диффузии компоненты $B$ соответственно в жидкой и твердой фазах, мобильность фазового поля выражена через параметр $v[28,30]$ :

$$
M_{\varphi}=\frac{v}{2 \sigma \delta} .
$$

Для перехода к безразмерным переменным в уравнениях заменим $x \rightarrow x / \delta$ и $t \rightarrow t v / \delta^{2}$. В этом случае уравнение для фазового поля в одномерном случае будет иметь вид [19]

$$
\begin{aligned}
\widetilde{\tau}_{\varphi} \frac{\partial^{2} \varphi}{\partial t^{2}}+\frac{\partial \varphi}{\partial t}= & \frac{\partial^{2} \varphi}{\partial x^{2}}-\frac{9}{2} \frac{\partial g(\varphi)}{\partial \varphi} \\
& +\frac{1}{2} \frac{\delta}{d_{0}} \frac{T}{T_{A}} \lambda(T, C, \varphi) \frac{\partial p(\varphi)}{\partial \varphi}
\end{aligned}
$$


в котором введено обозначение

$$
\lambda(T, C, \varphi)=\frac{\left(1-k_{e}\right) C}{k_{e}+\left(1-k_{e}\right) p(\varphi)}-\frac{1-k_{e}}{m_{e}}\left(T-T_{A}\right),
$$

где $\widetilde{\tau_{\varphi}}=\tau_{\varphi} v / \delta^{2}-$ безразмерное время релаксации скорости изменения фазового поля, $d_{0}=\sigma v_{m} /\left(R T_{A}\right)-$ капиллярная длина.

Безразмерное уравнение в частных производных для концентрации запишется как [19]

$$
\begin{gathered}
\widetilde{\tau}_{D} \frac{\partial^{2} C}{\partial t^{2}}+\frac{\partial C}{\partial t}=\frac{\partial}{\partial x}\left(\widetilde{D}(\varphi) \frac{\partial C}{\partial x}\right) \\
+\frac{\partial}{\partial x}\left(\Theta(T) \widetilde{D}(\varphi) C \frac{\partial p(\varphi)}{\partial \varphi} \frac{\partial \varphi}{\partial x}\right),
\end{gathered}
$$

где введены обозначения

$$
\Theta(T)=-\frac{1-k_{e}}{k_{e}+\left(1-k_{e}\right) p(\varphi)},
$$

$\widetilde{\tau}_{D}=v^{2} / D_{L} \delta^{2}-$ безразмерное время релаксации потока примеси, $v_{0}=v / D_{L}$. Безразмерный коэффициент диффузии будет иметь вид

$$
\widetilde{D}(\varphi)=\frac{D_{S}}{D_{L}}+p(\varphi)\left(1-\frac{D_{S}}{D_{L}}\right) .
$$

\section{3. Градиентно-устойчивые численные схемы для EFKР-модели}

Для численного решения гиперболических уравнений с учетом того, что фазовое поле $\varphi$ является несохраняющейся, а концентрация $C-$ сохраняющейся величиной, введем соответствующие „потоки“. Для этого формально обозначим „поток для фазового поля“ $u=\partial \varphi / \partial t$, как а для концентрации используем закон сохранения примеси с диффузионным потоком $J$ :

$$
\frac{\partial C}{\partial t}=-\nabla \cdot J
$$

В этом случае уравнения второго порядка по времени сводятся к уравнениям первого порядка, для которых в одномерном случае могут быть записаны полунеявные градиентно-устойчивые разностные схемы [35,36], имеющие следующий вид:

— для фазового поля

$$
\begin{gathered}
u_{i}^{k+1}=\frac{2 \widetilde{\tau}_{\varphi}-\Delta t}{2 \widetilde{\tau}_{\varphi}+\Delta t} u_{i}^{k}+\frac{2 \Delta t}{2 \widetilde{\tau}_{\varphi}+\Delta t}\left(\frac{\varphi_{i+1}^{k}+\varphi_{i-1}^{k}-2 \varphi_{i}^{k}}{\Delta x^{2}}\right) \\
-\frac{2 \Delta t}{2 \widetilde{\tau}_{\varphi}+\Delta t}\left(\frac{9}{2} \frac{\partial g\left(\varphi_{i}^{k}\right)}{\partial \varphi_{i}^{k}}+\lambda\left(C_{i}^{k}, \varphi_{i}^{k}\right) \frac{\partial p\left(\varphi_{i}^{k}\right)}{\partial \varphi_{i}^{k}}\right), \\
\varphi_{i}^{k+1}=\varphi_{i}^{k}+\frac{1}{2} \Delta t\left(u_{i}^{k+1}+u_{i}^{k}\right) ;
\end{gathered}
$$

— для концентрационного поля

$$
\begin{gathered}
J_{i}^{k+1}=\frac{2 \widetilde{\tau}_{D}-\Delta t}{2 \widetilde{\tau}_{D}+\Delta t} J_{i}^{k}+\frac{\Delta t}{2 \widetilde{\tau}_{D}+\Delta t} D\left(\varphi_{i}^{k}\right) \\
\times\left[\frac{C_{i+1}^{k}-C_{i-1}^{k}}{\Delta x}+\Theta\left(\varphi_{i}^{k}, C_{i}^{k}\right) \frac{\varphi_{i+1}^{k}-\varphi_{i-1}^{k}}{\Delta x}\right], \\
C_{i}^{k+1}=C_{i}^{k}+\frac{1}{2} \frac{\Delta t}{\Delta x}\left(J_{i}^{k+1}+J_{i}^{k}\right) .
\end{gathered}
$$

В формулах (П14)-(П17) величина $\Delta t$ является шагом по времени, $\Delta x-$ шаг по пространству, индекс $k$ нумерует временные узлы, индекс $i$ - пространственные узлы расчетной сетки.

Вычислительный алгоритм (П14)-(П17) имеет первый порядок точности по времени. Градиентная устойчивость алгоритма означает, что свободная энергия дискретного решения не возрастает при переходе от одного временного шага к другому. Чтобы получить явную разностную схему второго порядка по времени для алгоритма (П14)-(П17), используем модифицированную схему Эйлера. Для этого на этапе прогноза интерполяция (П14)-(П17) вычисляется с половинным шагом по времени $\Delta t / 2$ в результате чего находятся значения функций на промежуточном слое по времени. Далее значения функций на промежуточном шаге по времени применяются для вычисления правых частей уравнений (П14)-(П17). Повторное использование схемы (П14)-(П17), но уже с шагом $\Delta t$ и правой частью, вычисленной на промежуточном слое по времени, определяет функции на новом слое по времени, что в итоге дает разностную схему второго порядка точности по времени. Дальнейшее повышение точности алгоритма по времени не имеет особого смысла из-за нелинейности задачи.

Для расчета методом конечных разностей использовалась пространственная область размером $100 \delta$ разбиваемая на $N=4000$ интервалов (пространственных шагов). Данное разбиение выбрано как оптимальное по точности и затратам времени, что определялось из численных экспериментов с различными разбиениями. Поскольку схемы (П14)-(П17) явные, для них экспериментально получено ограничение шага по времени - численный алгоритм является условно устойчивым при выборе шага интегрирования по времени $\Delta t \leq 8.95 \Delta x^{2}$. Для сравнения сошлемся на аналитическое исследование устойчивости явной разностной схемы для уравнения локальнонеравновесной диффузии, проводившееся в работе [44], где получено достаточное условие устойчивости в виде $\Delta t \leq q \Delta x^{2} / 8$, где $q=\delta^{2} /\left(D_{L} \tau_{D}\right) \approx 9.77$. В результате полученное в [44] ограничение может быть представлено в виде $\Delta t \leq 1.22 \Delta x^{2}$. Отличие между экспериментально определенным условием и оценкой [44] обусловлено разницей между явной и градиентно-устойчивой схемой, использованной в настоящей работе. 


\section{Финансирование работы}

Исследование выполнено при финансовой поддержке РФФИ и Правительства Удмуртской Республики в рамках научного проекта № 18-42-180002, а также при частичной поддержке по проекту РФФИ № 18-02-00643 и German Science Foundation (DFG Deutsche Forshungsgemeinschaft) under the Project GA 1142/11-1.

\section{Конфликт интересов}

Авторы заявляют, что у них нет конфликта интересов.

\section{Список литературы}

[1] M.C. Flemings. Solidification Processing (Mc Graw-Hill book comp., NY., 1974)

[2] W. Kurz, D.J. Fisher. Fundamentals of Solidification (Trans Tech Pub., Lausanne, 1984)

[3] J.A. Danzig, M. Rappaz. Solidification (EPFL Press, Lausanne, 2009)

[4] A. Paolini, S. Kollmannsberger, E. Rank. Additive Manufacturing, 30, 100894 (2019). DOI: doi.org/10.1016/j.addma.2019.100894

[5] Электронный ресурс. Режим доступа: https://www.magmasoft.de, http://www.mkmsoft.ru, https://www.esi-group.com/products/casting from 28.08.2020

[6] Электронный ресурс. Режим доступа: https://micress.rwth-aachen.de from 28.08.2020

[7] I. Steinbach, L. Zhang, M. Plapp. Acta Mater., 60, 2689 (2012). DOI: 10.1016/j.actamat.2012.01.035

[8] H.F. Wang, P.K. Galenko, X. Zhang, W.W. Kuang, F. Liu, D.M. Herlach. Acta Mater., 90, 982 (2015). DOI: dx.doi.org/10.1016/j.actamat.2015.02.021

[9] В.Г. Лебедев, П.К. Галенко. Расплавы, 5, 422 (2016). [V.G. Lebedev, P.K. Galenko. Russ. Metall. (Metally), 8, 785 (2016). DOI: 10.1134/S0036029516080097]

[10] G. Boussinot, M. Apel, J. Zielinski, U. Hecht, J.H. Schleifenbaum. Phys. Rev. Appl., 11, 014025 (2019). DOI:doi.org/10.1103/PhysRevApplied.11.014025

[11] K. Karayagiz, L. Johnson, R. Seede, V. Attari, B. Zhang, X. Huang, S. Ghosh, T. Duong, I. Karaman, A. Elwany, R. Arroyave. Acta Mater., 185, 320 (2020). DOI: $10.1016 /$ j.actamat.2019.11.057

[12] C. Kumara, A. Segerstark, F. Hanning, N. Dixit, S. Joshi, J. Moverare, P. Nylén. Additive Manufacturing, 25, 357 (2019). DOI: doi.org/10.1016/j.addma.2018.11.024

[13] P. Galenko, S. Sobolev. Phys. Rev. E, 55, 343 (1997). DOI: doi.org/10.1103/PhysRevE.55.343

[14] P. Galenko, D. Danilov. J. Cryst. Growth, 197 (4), 992 (1999). DOI: doi.org/10.1016/S0022-0248(98)00977-4

[15] Y. Yang, H. Humadi, D. Buta, B.B. Laird, D. Sun, J.J. Hoyt, M. Asta. Phys. Rev. Lett., 107, 025505 (2011). DOI: doi.org/10.1103/PhysRevLett.107.025505

[16] N. Provatas, K. Elder. Phase-Field Methods in Materials Science and Engineering (Wiley-VCH, Weinheim, 2010)

[17] B. Echebarria, R. Folch, A. Karma, M. Plapp. Phys. Rev. E, 70, 061604 (2004). DOI: doi.org/10.1103/PhysRevE.70.061604

[18] V.G. Lebedev, E.V. Abramova, D.A. Danilov, P.K. Galenko. Int. J. Mater. Res., 101 (4), 473 (2010). DOI: doi.org/10.3139/146.110297
[19] P.K. Galenko, E.V. Abramova, D. Jou, D.A. Danilov, V.G. Lebedev, D.M. Herlach. Phys. Rev. E, 84, 041143 (2011). DOI: doi.org/10.1103/PhysRevE.84.041143

[20] J.C. Baker, J.W. Cahn. Acta Metall., 17, 575 (1969).

[21] D. Herlach, P. Galenko, D. Holland-Moritz. Metastable Solids from Undercooled Melts (Elsevier, Amsterdam, 2007)

[22] M.J. Aziz. J. Appl. Phys., 53, 1158 (1982).

[23] M.J. Aziz, T. Kaplan. Acta Metall., 36, 2335 (1988).

[24] J.A. Kittl, M.J. Aziz, D.P. Brunco, M.O. Thompson. J. Cryst. Growth, 148, 172 (1995). DOI: doi.org/10.1016/0022-0248(94)00836-1

[25] A.A. Wheeler, W.J. Boettinger, G.B. Mc Fadden. Phys. Rev. A, 45, 7424 (1992). DOI: doi.org/10.1103/PhysRevA.45.7424

[26] A.A. Wheeler, W.J. Boettinger, G.B. Mc Fadden. Phys. Rev. E, 47, 3, 1893 (1993).

[27] N.A. Ahmad, A.A. Wheeler, W.J. Boettinger, G.B. Mc Fadden. Phys. Rev. E, 58, 3436 (1998). DOI: doi.org/10.1103/PhysRevE.58.3436

[28] D. Danilov, B. Nestler. Discrete Contin. Dyn. Syst., 15, 1035 (2006). DOI: 10.3934/dcds.2006.15.1035

[29] P. Galenko. Phys. Rev. E, 76, 031606 (2007). DOI:doi.org/10.1103/PhysRevE.76.031606

[30] D. Danilov, B. Nestler, M. Guerdane, H. Teichler. J. Phys. D: Appl. Phys., 42, 015310 (2009). DOI: $10.1088 / 0022-3727 / 42 / 1 / 015310$

[31] M. Guerdane, F. Wendler, D. Danilov, H. Teichler, B. Nestler. Phys. Rev. B, 81, 224108 (2010). DOI: doi.org/10.1103/PhysRevB.81.224108

[32] D. Danilov, B. Nestler. Acta Mater., 54, 4659 (2006). DOI: doi.org/10.1016/j.actamat.2006.05.045

[33] P. Galenko. Phys. Lett. A, 287, 190 (2001). DOI: doi.org/10.1016/S0375-9601(01)00489-3

[34] P. Galenko, D. Jou. Phys. Rev. E, 71, 046125 (2005). DOI: doi.org/10.1103/PhysRevE.71.046125

[35] V. Lebedev, A. Sysoeva, P. Galenko. Phys. Rev. E, 83, 026705 (2011). DOI: 10.1103/PhysRevE.83.026705

[36] P. Galenko, V. Lebedev, A. Sysoeva. Comput. Math. Math. Phys., 51 (6), 1074 (2011). DOI: $10.1134 / \mathrm{s} 0965542511060078$

[37] A. Karma. Phys. Rev. Lett., 87, 115701 (2001). DOI:doi.org/10.1103/PhysRevLett.87.115701

[38] R.F. Almgren. SIAM J. Appl. Math., 59, 2086 (1999).

[39] T. Pinomaa, N. Provatas. Acta Mater., 168, 167 (2019). DOI: 10.1016/j.actamat.2019.02.009

[40] T. Pinomaa, M. Lindroos, M. Walbrühl, N. Provatas, A. Laukkanen. Acta Mater., 184, 1 (2020). DOI: doi.org/10.1016/j.actamat.2019.10.044

[41] A. Salhoumi, P.K. Galenko. Physica A, 447, 161 (2016). DOI: $10.1016 /$ j.physa.2015.12.042

[42] A. Salhoumi, P.K. Galenko. IOP Conf. Series: Mat. Sci. and Eng., 192, 012014 (2017). DOI: $10.1088 / 1757-899 \mathrm{X} / 192 / 1 / 012014$

[43] D. Kessler. J. Cryst. Growth, $224(1-2), 175$ (2001). DOI: 10.1016/S0022-0248(01)00814-4

[44] P.K. Galenko, M.D. Krivilyov. Model. Simul. Mater. Sci. Eng., 8, 67 (2000). DOI: doi.org/10.1088/0965-0393/8/1/307 